\title{
QUASI-PROJECTIVE COVERS AND DIRECT SUMS
}

\author{
ANNE KOEHLER
}

\begin{abstract}
In this paper $R$ denotes an associative ring with an identity, and all modules are unital left $R$-modules. It is shown that the existence of a quasi-projective cover for each module implies that each module has a projective cover. By a similar technique the following statements are shown to be equivalent: $1 . R$ is semisimple and Artinian; 2. Every finitely generated module is quasi-projective; and 3. The direct sum of every pair of quasiprojective modules is quasi-projective. Direct sums of quasiinjective modules are also investigated.
\end{abstract}

1. Quasi-projective covers. Rings which have a projective cover for each module are called left perfect rings, and such rings have been characterized by $\mathrm{H}$. Bass [1]. A module $M$ has a projective cover $P(M)$ iff there is an epimorphism $\phi: P(M) \rightarrow M$ such that $P(M)$ is a projective module, and $\operatorname{Ker} \phi$ is small in $P(M)$ (i.e. $A=P$ whenever $A$ $+\operatorname{Ker} \phi=P(M))$. A module is quasi-projective iff for every epimorphism $q: M \rightarrow A, \operatorname{Hom}(M, A)=q \circ \operatorname{Hom}(M, M)[8]$ and [11]. Wu and Jans have defined a quasi-projective cover as follows: $Q P(M)$ is a quasi-projective cover of $M$ iff there exists an epimorphism $\phi: Q P(M)$ $\rightarrow M$ such that 1. $Q P(M)$ is quasi-projective, 2 . Ker $\phi$ is small in $Q P(M)$, and 3. if $0 \neq B \subseteq \operatorname{Ker} \phi$, then $Q P(M) / B$ is not quasi-projective. They show that if a module has a projective cover, then it must have a quasi-projective cover. The converse to this theorem is false. The question of what can be said if each module has a quasi-projective cover is answered by the first theorem.

THEOREM 1.1. If every module has a quasi-projective cover, then every module has a projective cover.

Proof. Let $M$ be an arbitrary module and $R^{M}$ be the direct sum of $\operatorname{card}(M)$ copies of $R$. There is an epimorphism $\phi: R^{M} \rightarrow M$. Let $\pi: Q$ $\rightarrow R^{M} \oplus M$ be a quasi-projective cover of $R^{M} \oplus M$ and $q: R^{M} \oplus M \rightarrow R^{M}$ be the usual projection map. Since $R^{M}$ is projective, there is a monomorphism $i: R^{M} \rightarrow Q$ such that $q \circ \pi \circ i=$ identity on $R^{M}$, and $Q$ $=\operatorname{Im}(i) \oplus \operatorname{Ker}(q \circ \pi)$. Let $\bar{M}=\operatorname{Ker}(q \circ \pi)$ and $\pi_{1}=\left.\pi\right|_{\bar{M}}$. Then we can assume $Q=R^{M} \oplus \bar{M}$. We claim that $\bar{M}$ is the projective cover of $M$ with the desired epimorphism being $\pi_{1}$. Suppose $\operatorname{Ker} \pi_{1}+A=\bar{M}$. Since Ker $\pi_{1} \subseteq \operatorname{Ker} \pi$ and $R^{M} \oplus \operatorname{Ker} \pi_{1}+A=R^{M} \oplus \bar{M}=Q$, we have

Received by the editors July 25, 1969.

AMS Subject Classifications. Primary 1640, 1690, 1644.

Key Words and Phrases. Quasi-projective module, projective cover, perfect ring, quasi-projective cover, quasi-injective module, semisimple ring. 
$R^{M} \oplus A=R^{M} \oplus \bar{M}$ or $A=\bar{M}$. Thus Ker $\pi_{1}$ is small in $\bar{M}$.

Now we need to show that $\bar{M}$ is projective. Let $q^{\prime}: R^{M} \oplus \bar{M} \rightarrow \bar{M}$ be the projection onto $\bar{M}$ and $j: \bar{M} \rightarrow R^{M} \oplus \bar{M}$ be the monomorphism such that $q^{\prime} \circ j=$ id. $R^{M}$ is projective, so there is a homomorphism $\phi^{\prime}: R^{M}$ $\rightarrow \bar{M}$ such that

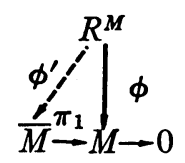

commutes. The homomorphism $\phi^{\prime}$ is onto because $\operatorname{Ker} \pi_{1}$ is small. Since $R^{M} \oplus \bar{M}$ is quasi-projective there is an $h \in \operatorname{End}_{R}\left(R^{M} \oplus \bar{M}\right)$ such that

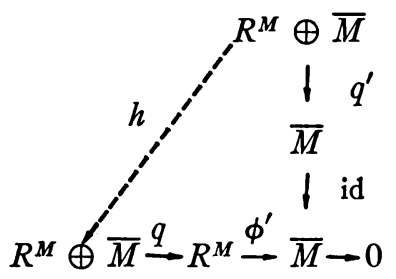

commutes, that is, $\phi^{\prime} \circ q \circ h=$ id $\circ q^{\prime}$. Let $f=q \circ h \circ j$. Then $\phi^{\prime} \circ f$ $=\mathrm{id}$, and $\bar{M}$ is isomorphic to a direct summand of $R^{M}$. Hence $\bar{M}$ is the projective cover of $M$.

COROLlARY 1.2. Every module has a quasi-projective cover iff $R$ is left perfect.

COROLlary 1.3. A ring $R$ is semiperfect [1] iff every finitely generated module has a quasi-projective cover.

REMARK. It should be noted that property 3 in the definition of a quasi-projective cover was not needed for the proof of Theorem 1.1.

2. Direct sums. Direct sums of quasi-projective (quasi-injective) modules do not need to be quasi-projective (quasi-injective). Necessary and sufficient conditions for these direct sums to be quasiprojective (quasi-injective) will now be studied. A module $M$ is quasiinjective iff for every monomorphism $i: A \rightarrow M, \operatorname{Hom}(A, M)$ $=\operatorname{Hom}(M, M) \circ i$.

THEOREM 2.1. The following statements are equivalent:

1. $R$ is semisimple and Artinian;

2. Every finitely generated module is quasi-projective; and

3. The direct sum of two quasi-projective modules is always quasiprojective. 
Proof. It is known that 1 implies every module is projective. Thus 1 implies 2 and 3 . It is also known that if every simple module is projective, then $R$ is semisimple and Artinian. ${ }^{1}$ We will show that $R$ is semisimple and Artinian if $R \oplus M$ is quasi-projective for every simple module $M$. It follows from this result that 2 implies 1 and 3 implies 1. Assume $M$ is simple. Then there is an epimorphism $\phi: R$ $\rightarrow M$. Let $q_{1}: R \oplus M \rightarrow M$ be the projection of $R \oplus M$ onto $M$ and $q_{2}: R \oplus M \rightarrow R$ be the projection onto $R$. Let $i: M \rightarrow R \oplus M$ be the monomorphism such that $q_{1} \circ i=\mathrm{id}$. If $R \oplus M$ is quasi-injective then then there is an $h \in \operatorname{End}(R \oplus M)$ such that

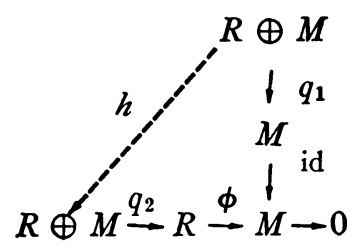

commutes. Let $f=q_{2} \circ h \circ i$. Then $\phi \circ f=\mathrm{id}$, and $M$ is isomorphic to a direct summand of $R$. Thus every simple module is projective.

THEOREM 2.2. If the direct sum of two quasi-injective modules is always quasi-injective, then every quasi-injective module is injective.

Proof. Let $M$ be a quasi-injective module and $Q$ be its injective hull. There is a monomorphism $i: M \rightarrow Q$. Let $j_{1}: M \rightarrow Q \oplus M$ be the injection of $M$ into $Q \oplus M$ and $j_{2}: Q \rightarrow Q \oplus M$ be the injection of $Q$. Let $q: Q \oplus M \rightarrow M$ be the epimorphism such that $q \circ j_{1}=$ id. Since $Q \oplus M$ is quasi-injective, there is a $g \in \operatorname{End}(Q \oplus M)$ such that

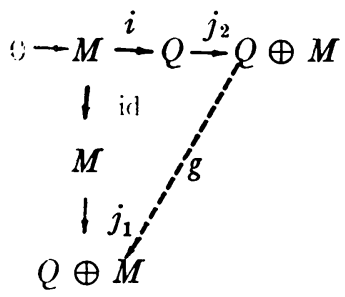

commutes. Let $f=q \circ g \circ j_{2}$. Then $f \circ i=\mathrm{id}$, and hence, $M$ is isomorphic to a direct summand of $Q$. Therefore $M$ is injective.

CoROllary 2.3. If the direct sum of every two quasi-injective modules is quasi-injective, then $R$ is semisimple and Noetherian.

1 This result was proved by G. Azumaya for a class in 1965 but is unpublished. See [10] for a published proof that $R$ is semisimple if every maximal right ideal is a direct summand of $R$. 
Proof. Since completely reducible modules are quasi-injective, they are injective. In particular every simple module is injective. Beachy has shown in this case that every proper ideal is the intersection of maximal ideals. Thus $R$ is semisimple. Kurshan has proved that if direct sums of injective hulls of simple modules are injective, then $R$ is Noetherian [6]. Therefore, $R$ is Noetherian in the present case.

Corollary 2.4. A ring $R$ is self-injective, and the direct sum of every pair of quasi-injective modules is quasi-injective iff $R$ is semisimple and Artinian.

Proof. It is known that if $R$ is injective and Noetherian, then $R$ is Artinian [4]. So by Corollary 2.3 the "only if" direction is proved. The converse is true because every module is injective if $R$ is Artinian and semisimple.

REMARKs. 1. Corollary 2.4 strengthens a result of Chaptal [2].

2. If the conjecture that a ring is von Neumann regular if every simple module is injective is true, then one would not need to assume $R$ is injective in Corollary 2.4. This result is true when $R$ is commutative [9].

\section{REFERENCES}

1. H. Bass, Finitistic dimension and a homological generalization of semi-primary rings, Trans. Amer. Math. Soc. 95 (1960), 466-488. MR 28 \#1212.

2. N. Chaptal, Sur les modules quasi-injectifs, C. R. Acad. Sci. Paris Sér. A-B 264 (1967), A173-A175. MR 34 \#7574.

3. J. Beachy, Some homological classes of rings and modules, Ph.D. Thesis, Indiana University, Bloomington, Ind., 1967.

4. C. Faith Rings with ascending condition on annihilators, Nagoya Math. J. 27 (1966), 179-191. MR 33 \#1328.

5. C. Faith and Y. Utumi, Quasi-injective modules and their endomorphism rings, Arch. Math. 15 (1964), 166-174. MR 29 \#3503.

6. R. P. Kurshan, Rings whose cyclic modules have finitely generated socle, (to appear).

7. J. Lambek, Lectures on rings and modules, Blaisdell, Waltham, Mass., 1966. MR $34 \# 5857$.

8. Y. Miyashita, Quasi-projective modules, perfect modules, and a theorem for modular lattices, J. Fac. Sci. Hokkaido Univ. Ser I 19 (1966), 86-110. MR 35 \#4254.

9. A. Rosenberg and D. Zelinsky, Finiteness of the injective hull, Math. Z. 70 (1958/59), 372-380. MR 21 \#4176.

10. M. Satyanarayana, Semisimple rings, Amer. Math. Monthly 74 (1967), 1086. MR 37 \#1403.

11. L. E. T. Wu and J. P. Jans, On quasi projectives, Illinois J. Math. 11 (1967), 439-448. MR $36 \# 3817$.

Miami University, OXFord, OHIo 